\title{
EXTRACTORES QUÍMICOS EN LA DETERMINACION DE SILICIO DISPONIBLE EN SUELOS ÁCIDOS, NEUTROS Y ALCALINOS
}

\section{CHEMICAL EXTRACTANTS IN THE EVALUATION OF AVAILABLE SILICON IN ACID SOILS, NEUTRAL AND ALKALINE}

\author{
*Enrique Combatt C. ${ }^{1}$, Daniel Palacio B. ${ }^{2}$, Jaime Mercado L. ${ }^{3}$
}

\begin{abstract}
${ }^{1}$ I.A. Ph.D., Departamento de Ingeniería Agronómica y Desarrollo Rural, Facultad de Ciencias Agrícolas. Universidad de Córdoba, carrera 6 No 76-103, Montería-Córdoba, Colombia, e-mail: *ecombatt@correo.unicordoba.edu.co; ${ }^{2}$ Químico, auxiliar de laboratorio. Carrera 6 No 76-103, Montería-Córdoba, Colombia; ${ }^{3}$ Químico, M.Sc, Coordinador analítico del laboratorio de suelos. Universidad de Córdoba, carrera 6 No 76-103, Montería-Córdoba, Colombia
\end{abstract}

Rev. U.D.C.A Act. \& Div. Cient. 20(2): 329-339, Julio-Diciembre, 2017

\section{RESUMEN}

El contenido de silicio disponible, generalmente, se ha determinado en suelos ácidos, lo que ha descuidado su cuantificación en otros suelos, para poder realizar recomendaciones de este elemento, acorde a su contenido para cada suelo. El objetivo fue evaluar el silicio disponible por diferentes extractores químicos en suelos del departamento de Córdoba, en Colombia. Para ello, se colectaron, a la profundidad de $20 \mathrm{~cm}$, muestras de suelos ácidos, neutros y alcalinos, que fueron ingresados al laboratorio de Suelos y Aguas de la Universidad de Córdoba, para determinar el contenido de silicio disponible. Los extractores químicos fueron cloruro de calcio $0,01 \mathrm{~mol} \mathrm{~L}^{-1}$, ácido acético $0,5 \mathrm{~mol} \mathrm{~L}^{-1}$, acetato de amonio 38,5 $\mathrm{L} \mathrm{L}^{-1}$, tampón pH 4,0 $\left(\mathrm{HC}_{2} \mathrm{H}_{3} \mathrm{O}_{2}+\mathrm{CH}_{3} \mathrm{COONa}\right)$ y el método propuesto por los autores, denominado solución de cloruro de bario. Para la extracción, se pesaron $5 \mathrm{~g}$ de suelo y se le adicionó $50 \mathrm{~mL}$ de solución extractante y la mezcla fue agitada durante $1 \mathrm{~h}$, a 220rpm, filtrada y dejada en reposo por $12 \mathrm{~h}$. La cuantificación, se realizó en un espectrofotómetro, a una longitud de onda de 66nm y la determinación química, se efectuó en serie, con tres repeticiones, las cuales, fueron analizadas por correlación y contrastes. En general, de acuerdo a los resultados de mayor a menor concentración de silicio, se presentó en la siguiente secuencia de extracción, suelos alcalinos > neutros > ácidos. Mediante las pruebas estadísticas de Tukey y contrastes, se concluyó que el método solución de cloruro de bario se puede utilizar como alternativo al método de referencia en la determinación de silicio en los tres tipos de suelos. Además, para suelos alcalinos, se puede emplear el método de acetato de amonio $38,5 \mathrm{~g} \mathrm{~L}^{-1}$, como alternativa para la determinación de silicio.
Palabras clave: Métodos químicos; reactivos; metodologías analíticas; elemento químico.

\section{SUMMARY}

The available silicon content has generally been determined in acid soils, but neglected its quantification in other soils, to be able to make recommendations of this element according to its content for each soil. The objective of this research was to evaluate the available silicon in soils of the department of Córdoba, Colombia by means of different chemical extractors. Samples of acid, neutral and alkaline soils were collected at the depth of $20 \mathrm{~cm}$ and sent to the Laboratory of Soils and Waters of the University of Córdoba to determine the available silicon content. The chemical extractors were $0.01 \mathrm{~mol} \mathrm{~L}^{-1}$ calcium chloride, $0.5 \mathrm{~mol} \mathrm{~L}^{-1}$ acetic acid, $38.5 \mathrm{~g} \mathrm{~L}^{-1}$ ammonium acetate, $\mathrm{pH} 4.0$ buffer $\left(\mathrm{HC}_{2} \mathrm{H}_{3} \mathrm{O}_{2}+\mathrm{CH}_{3} \mathrm{COONa}\right)$ and the method proposed by the authors, named barium chloride solution. For extraction, $5 \mathrm{~g}$ of soil were weighed and $50 \mathrm{~mL}$ of extractant was added and the mixture was stirred for $1 \mathrm{~h}$ at 220rpm, filtered and left standing for $12 \mathrm{~h}$. The quantification was performed in a spectrophotometer at a wavelength of $66 \mathrm{~nm}$ and the chemical determination was performed in series with three replicates, analyzed by correlation and contrasts. In general, according to results of higher to lower concentration, silicon was presented in the following sequence of extraction, alkaline soils $>$ neutral $>$ acids. By means of the Tukey test and contrasts it was concluded that the barium chloride solution could be used as alternative to the reference method in the determination of silicon in the three types of soils. In addition, for alkaline soils, the ammonium acetate method $38.5 \mathrm{~g} \mathrm{~L}^{-1}$ can also be used as an alternative for the determination of silicon. 
Key words: Chemical methods; reagents; analytical methodologies; chemical element.

\section{INTRODUCCIÓN}

El silicio es un elemento esencial y benéfico para cultivos acumuladores de silicio, como arroz, caña de azúcar, cebada, maíz y trigo, que se siembran en suelos ácidos, neutros y alcalinos, en muchas regiones de mundo (Lima et al. 2011). Por lo tanto, es necesario implementar métodos o paquetes de métodos, que permitan un cambio crítico en el entendimiento de las fracciones funcionales hasta estructural y funcionalmente definidas de los elementos cuantificados (Condron \& Newman, 2011).

En Colombia, son pocos los estudios que se han realizado para determinar la concentración de este elemento, por consiguiente, la aplicación, como nutriente esencial, no se tiene en cuenta en el desarrollo de los cultivos, además la falta de investigación sobre la extracción, la cuantificación y la calibración de métodos analíticos, ha ocasionado el poco interés de productores e investigadores a estudiar las funciones de este elemento, en la nutrición de cultivos. Pulz et al. (2008) indican que los efectos benéficos del silicio han sido demostrados en varias especies vegetales, especialmente, cuando las plantas son sometidas a algún tipo de estrés, ya sea de carácter biótico o abiótico. Según Shetty et al. (2011), el efecto beneficioso de Si se ha demostrado, cuando las plantas sometidas a ataque de enfermedades y algunos insectos, los cultivos incrementan la actividad de enzimas involucradas en la defensa de las plantas, tales como peroxidasa, polifenoloxidasa, fenilalanina amoniacasa y lipoxigenasa. Fauteux et al. (2005) expone que, en el caso de un ataque de hongos patógenos, se desencadena un rápido y amplio despliegue de las defensas naturales de la planta, ya sea mediante el secuestro de cationes o directamente mediante el aumento de cierta actividad de las proteínas. Asimismo, la mayoría de los laboratorios de suelos en Colombia no determinan el elemento en sus rutinas analíticas, porque es un elemento poco utilizado en la nutrición de cultivos.

La extracción química del silicio, como nutriente, consiste en poder estimar la disponibilidad de este elemento en suelos, que pueda ser absorbido por los cultivos, pero esta extracción debe correlacionar con la cantidad del elemento que es asimilado por un cultivo, bajo unas condiciones químicas determinadas. Según Havlin et al. (2005), el extractor desplaza e intercambia una parte de la cantidad total del elemento, que está relacionada con la cantidad absorbida por las plantas y este análisis de extracción deberá reproducir la cantidad del nutriente que se encuentra en el suelo, ya sea en la solución, en la fase intercambiable, orgánica o mineral que son utilizadas por las plantas.
Existen diversos extractores de silicio disponible que han sido utilizados en varias partes del mundo, por diferentes investigadores. Lima et al. (2003) concluyeron que el extractor de acetato de sodio tamponado a $\mathrm{pH} 4,0$, extrajo las mayores cantidades de Si y sobrestima la disponibilidad de silicio en el suelo. Snyder (2001) demostró que el extractor de ácido acético $0,5 \mathrm{~mol} \mathrm{~L}^{-1}$ es bastante práctico y eficiente, en identificar suelos con deficiencia de este elemento. Wang et al. (2004) indicaron que la acidez y los aniones podrían impactar de forma aditiva la liberación de Si de los suelos. Por su parte Lima et al. (2003) expone que las soluciones más eficientes son las ácidas en comparación con las soluciones neutras, debido a que los extractores ácidos sobreestiman los contenidos de silicio disponible y esto es relacionado con el pH de las soluciones extractoras.

Otro de los extractores que es utilizado actualmente es el cloruro de calcio $0,01 \mathrm{~mol} \mathrm{~L}^{-1}$, el cual, según Vidal (2003) presenta las mayores correlaciones entre el silicio extraído en el suelo y la cantidad de silicio absorbido por la planta. Según Korndörfer et al. (2004), el extractor de silicio más utilizando es el cloruro de calcio $0,01 \mathrm{~mol} \mathrm{~L}^{-1}$; sin embargo, se necesitan más estudios sobre diferentes extractores para los diferentes suelos, ya que los resultados de extracción son diferentes y dependen de las características físicas y químicas de los suelos. Además, Berthelsen \& Korndörfer (2005) indican que hay una gran diversidad de métodos de extracción de silicio disponible para las plantas y ningún extractor es igualmente eficaz, en todos los tipos de suelos, porque la cantidad extraída de silicio varía de acuerdo a la solución extractora y al tipo de suelo.

Como Córdoba, Colombia, es un departamento con una gran variedad de suelos y los estudios sobre la determinación de silicio disponible son escasos y su dinámica de extracción no es totalmente conocida, el objetivo de esta investigación fue evaluar la correlación que existe entre el método de referencia $\mathrm{CaCl}_{2}$,, $01 \mathrm{~mol} \mathrm{~L}^{-1}$ y cuatro extractores químicos de silicio disponible, entre los cuales, se encuentra la solución de cloruro de bario.

\section{MATERIALES Y MÉTODOS}

Este estudio fue realizado en el laboratorio de Suelos y Aguas de la Facultad de Ciencias Agrícolas de la Universidad de Córdoba. Fueron colectadas 18 muestras de suelo de $5 \mathrm{~kg}$, a una profundidad hasta $20 \mathrm{~cm}$, en zonas establecidas con pasto, donde seis fueron suelos ácidos (Municipio de Montelibano), seis neutros (Municipio de Montería Moñito) y seis alcalinos (Municipio de Moñito). Estas muestras de suelos se secaron a temperatura ambiente y fueron sometidas a trituración, en un molino eléctrico, marca Thurm TGL-8394 y, posteriormente, pasadas por malla de $2 \mathrm{~mm}$, en tamices Soils test ASTM E-11, para lograr su homogenización. Finalmente, las 
muestras fueron empacadas en recipientes plásticos, herméticamente cerrados, para evitar el exceso de humedad. Estas muestras fueron caracterizadas químicamente, de acuerdo a las metodologías del IGAC (2006), seguidas por el laboratorio, a excepción del boro disponible, que fue determinado

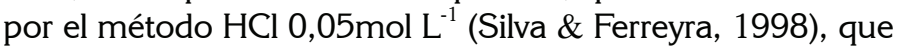
se observan en la tabla 1.

Métodos de extracción para la determinación de silicio disponible. Para cuantificar el contenido de silicio disponible en los suelos, se utilizaron cinco extractores, empleando una relación de $5 \mathrm{~g}$ de suelo, con $50 \mathrm{~mL}$ de solución extrac- tora, agitados por 1h, a 220rpm. Los métodos de extracción fueron cloruro de calcio $\left(\mathrm{CaCl}_{2}\right)$ 0,01 $\mathrm{mol} \mathrm{L}^{-1}$, denominado método de referencia, de acuerdo con Korndörfer et al. (2004); extracción con ácido acético $\left(\mathrm{HC}_{2} \mathrm{H}_{3} \mathrm{O}_{2}\right) 0,5 \mathrm{~mol} \mathrm{~L}^{-1}$ (Korndörfer et al. 2004); extracción con acetato de amonio $\left(\mathrm{CH}_{3} \mathrm{COONH}_{4}\right) 38,5 \mathrm{~g} \mathrm{~L}^{-1}$ (Pereira et al. 2007); extracción con tampón pH 4,0 (42mL $\mathrm{HC}_{2} \mathrm{H}_{3} \mathrm{O}_{2}+14,8 \mathrm{~g}$ de $\left.\mathrm{CH}_{3} \mathrm{COONa}\right)$ (Korndörfer et al. 1999) y, por último, la extracción de silicio en $5 \mathrm{~g}$ de suelo, con $49 \mathrm{~mL}$ de agua $+1 \mathrm{~mL}$ de $\mathrm{BaCl}_{2}$ al $10 \%$, denominada por los autores como solución de cloruro de bario, por introducir $\mathrm{BaCl}_{2}$ al $10 \%$, en la metodología de extracción solo con agua.

Tabla 1. Caracterización química de suelos ácidos, neutros y alcalinos, del departamento de Córdoba, Colombia.

\begin{tabular}{|c|c|c|c|c|c|c|c|c|c|c|c|c|c|c|c|}
\hline \multirow{2}{*}{ Suelos } & $\mathrm{pH}$ & MO & $\mathrm{s}$ & $\mathbf{P}$ & $\mathrm{Ca}$ & $\mathrm{Mg}$ & $\mathrm{K}$ & $\mathrm{Na}$ & $\mathrm{Al}+\mathrm{H}$ & CICE & $\mathrm{Cu}$ & $\mathrm{Fe}$ & $\mathrm{Zn}$ & Mn & B \\
\hline & $1: 1$ & $\%$ & \multicolumn{2}{|c|}{----mg kg ${ }^{-1}---$} & \multicolumn{6}{|c|}{----------------cmol ${ }^{+}$kg $^{-1}$---------------- } & \multicolumn{5}{|c|}{ 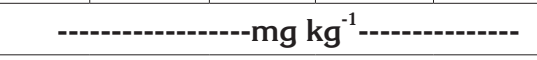 } \\
\hline & \multicolumn{15}{|c|}{ SUELOS ÁCIDOS } \\
\hline 1 & 4,24 & 2,07 & 23,80 & 2,40 & 4,50 & 4,40 & 0,27 & 0,20 & 5,20 & 14,47 & 4,60 & 50,40 & 3,90 & 14,40 & 0,12 \\
\hline 2 & 4,72 & 0,86 & 12,70 & 1,40 & 0,10 & 0,20 & 0,03 & 0,07 & 0,92 & 1,32 & 0,40 & 56,00 & 0,60 & 2,60 & 0,10 \\
\hline 3 & 5,35 & 0,17 & 7,00 & 0,20 & 3,50 & 3,90 & 0,10 & 0,15 & 4,22 & 11,87 & 1,00 & 30,40 & 0,50 & 4,40 & 0,08 \\
\hline 4 & 5,32 & 0,52 & 8,20 & 1,00 & 1,20 & 2,10 & 0,12 & 0,17 & 2,16 & 5,75 & 1,40 & 18,40 & 0,90 & 12,00 & 0,09 \\
\hline 5 & 5,94 & 1,03 & 13,60 & 2,20 & 4,50 & 1,40 & 0,17 & 0,22 & & 6,29 & 1,50 & 50,00 & 1,00 & 16,00 & 0,16 \\
\hline 6 & 5,83 & 0,34 & 7,90 & 3,00 & 1,50 & 0,70 & 0,05 & 0,10 & & 2,35 & 0,40 & 32,00 & 1,90 & 8,00 & 0,12 \\
\hline Promedio & 5,23 & 0,83 & 12,2 & 1,7 & 2,55 & 2,11 & 0,12 & 0,15 & 3,12 & 7,00 & 1,55 & 82,0 & 1,46 & 9,5 & 0,11 \\
\hline \multicolumn{16}{|c|}{ SUELOS NEUTROS } \\
\hline 1 & 6,30 & 1,71 & 97,20 & 3,80 & 22,50 & 6,90 & 0,37 & 0,23 & & 30 & 1,30 & 71,60 & 1,60 & 55,58 & 0,15 \\
\hline 2 & 6,42 & 0,51 & 20,50 & 7,20 & 18,40 & 6,80 & 0,22 & 0,23 & & 25,65 & 1,80 & 40,40 & 1,60 & 48,00 & 0,14 \\
\hline 3 & 5,97 & 1,87 & 8,40 & 2,20 & 14,70 & 15,80 & 0,22 & 0,36 & & 31,08 & 0,70 & 16,80 & 0,92 & 22,80 & 0,13 \\
\hline 4 & 6,74 & 2,73 & 15,50 & 9,40 & 47,50 & 7,20 & 0,41 & 0,98 & & 56,09 & 0,20 & 3,00 & 1,00 & 8,80 & 0,31 \\
\hline 5 & 6,17 & 1,20 & 7,60 & 3,80 & 6,80 & 3,20 & 0,11 & 0,53 & & 10,64 & 1,10 & 53,20 & 1,00 & 35,60 & 0,12 \\
\hline 6 & 6,56 & 3,10 & 7,00 & 5,30 & 21,60 & 18,30 & 0,47 & 0,48 & & 40,85 & 0,20 & 2,64 & 3,08 & 18,00 & 0,36 \\
\hline Promedio & 6,36 & 1,86 & 26,0 & 5,30 & 21,9 & 9,70 & 0,30 & 0,47 & & 32,3 & 0,89 & 31,2 & 1,53 & 31,4 & 0,20 \\
\hline \multicolumn{16}{|c|}{ SUELOS ALCALINOS } \\
\hline 1 & 7,35 & 0,17 & 13,0 & 44,22 & 19,00 & 15,00 & 0,14 & 1,17 & & 35,31 & 0,48 & 16,40 & 1,32 & 32,39 & 0,16 \\
\hline 2 & 8,08 & 1,03 & 7,00 & 87,10 & 37,00 & 7,50 & 0,49 & 0,33 & & 45,32 & 0,20 & 0,70 & 0,30 & 18,80 & 0,08 \\
\hline 3 & 8,21 & 0,34 & 7,00 & 55,90 & 38,50 & 10,00 & 0,20 & 0,28 & & 48,98 & 0,40 & 10,40 & 0,40 & 14,80 & 0,16 \\
\hline 4 & 7,39 & 1,21 & 7,30 & 32,10 & 26,50 & 24,10 & 0,25 & 0,83 & & 51,68 & 0,30 & 2,60 & 0,60 & 2,24 & 0,16 \\
\hline 5 & 8,11 & 0,17 & 8,40 & 44,40 & 46,50 & 1,70 & 0,14 & 0,15 & & 48,49 & 0,20 & 0,60 & 0,10 & 12,40 & 0,06 \\
\hline 6 & 7,81 & 2,23 & 7,02 & 16,60 & 36,00 & 2,60 & 0,29 & 0,13 & & 39,02 & 0,16 & 1,50 & 0,08 & 8,00 & 0,08 \\
\hline Promedio & 7,83 & 0,86 & 8,30 & 46,72 & 33,92 & 10,15 & 0,25 & 0,48 & & 44,80 & 0,28 & 5,37 & 0,46 & 14,77 & 0,12 \\
\hline
\end{tabular}

pH: Relación suelo-agua 1:1. MO: Walkley - Black. S: Fosfato de calcio monobásico 0,008 mol L ${ }^{-1}$. P: Bray -II modificado. Ca-Mg-K-Na: Acetato de amonio 1,0mol L ${ }^{-1} \mathrm{pH}$ 7,0, $\mathrm{Al}+\mathrm{H}: \mathrm{KCl}$ 1,0mol L ${ }^{-1}$. CICe: Suma de bases. Cu-Fe-Zn Mn: Mehlich - 1, B: $\mathrm{HCl} 0,05 \mathrm{~mol} \mathrm{~L}^{-1}$. 
Cuantificación del contenido de silicio disponible en los suelos. Después de agregar las soluciones extractoras por separado, denominadas como tratamientos, a cada muestra de suelo y una vez transcurrida una hora de agitación, se filtraron las soluciones en papel número 42 y los filtrados se dejaron en reposo, por un periodo de $12 \mathrm{~h}$. Para la cuantificación de silicio disponible, se colectó una alícuota de $10 \mathrm{~mL}$ del extracto filtrado de cada muestra y se colocó en un vaso plástico de $50 \mathrm{~mL}$; seguidamente, se adicionó al extracto $1 \mathrm{~mL}$ de solución sulfomolibdica, para la formación del complejo $\beta$-molibdosilicato de color amarillo, que ocurre después de $5 \mathrm{~min}$, a pH 1,4 y 2,0. A excepción de la extracción con $\mathrm{CH}_{3} \mathrm{COONH}_{4} 38,5 \mathrm{~g} \mathrm{~L}^{-1}$, al cual, hubo la necesidad de disminuir el pH de la solución antes de la aplicación de la solución sulfomolibdica, adicionándole $3 \mathrm{~mL}$ de ácido clorhídrico $1 \mathrm{~mol} \mathrm{~L}^{-1}$ en $10 \mathrm{~mL}$ de extracto. Después de transcurrido 1 min de la adición de la solución sulfomolibdica fueron adicionados $2 \mathrm{~mL}$ de ácido tartárico $200 \mathrm{~g} \mathrm{~L}^{-1}$ utilizado, para acomplejar el fósforo de la solución.

Seguidamente, después de $5 \mathrm{~min}$, a las concentraciones de la curva y de cada solución extraída por los métodos, fueron adicionados $10 \mathrm{~mL}$ de ácido ascórbico $3 \mathrm{~g} \mathrm{~L}^{-1}$, para que en la reducción del complejo $\beta$-molibdosilicato de color amarrillo, pasara a azul de molibdeno y después de una $1 \mathrm{~h}$, se realizó la lectura, en un espectrofotómetro Perkim Elmer lambda $\mathrm{XLS}+$ a $660 \mathrm{~nm}$.

Para la extracción del silicio, se utilizaron 3 repeticiones, que fueron analizadas en bloque, denominadas como series en la realización de los análisis químicos. Los resultados de silicio disponible extraído por los métodos de extracción en los suelos evaluados fueron sometidos a correlaciones, pruebas de medias por la prueba de tukey y contrastes, a un nivel del $5 \%$ de probabilidad, en el Software estadístico SAS (Statistical Analysis System) versión 8.0.

\section{RESULTADOS Y DISCUSIÓN}

La caracterización de los parámetros químicos de los suelos ratificó que presentaron características de suelos ácidos, neutros y alcalinos (Tabla 1). Los suelos ácidos expusieron, en promedio, un $\mathrm{pH}$ de 5,23; bajos contenidos de bases, alta concentración de acidez intercambiable, de $3,12 \mathrm{cmol}^{+} \mathrm{kg}^{-1} \mathrm{y}$ entre los elementos menores el hierro presentó los mayores contenidos $82,03 \mathrm{mg} \mathrm{kg}^{-1}$. Para los suelos neutros, se observa que los contenidos de calcio y de magnesio aumentaron con respecto a los suelos ácidos, con promedio de 21,92 y $9,70 \mathrm{mg} \mathrm{kg}^{-1}$, respectivamente. Estos suelos, se caracterizaron por poseer un $\mathrm{pH}$ entre 5,97 y 6,74, contenidos medios de hierro de $31,2 \mathrm{mg} \mathrm{kg}^{-1}$ y contenidos bajos de fósforo, con $5,30 \mathrm{mg} \mathrm{kg}^{-1}$. Para los suelos alcalinos, un pH de 7,83; bajo porcentaje de materia orgánica de $0,86 \%$ y bajos contenidos de fósforo, con $5,3 \mathrm{mg} \mathrm{kg}^{-1}$; contenido de calcio y de magne- sio en promedio de 33,92 y $10,15 \mathrm{cmol}^{+} \mathrm{kg}^{-1}$, alta capacidad de intercambio catiónico, con $44,8 \mathrm{cmol}^{+} \mathrm{kg}^{-1}$ y bajas concentraciones de elementos menores (Tabla 1).

Extracción de silicio en suelos ácidos, neutros y alcalinos. En estos suelos fue determinado que la extracción de silicio disponible en suelos ácidos de mayor a menor concentración se presentó en el siguiente orden: $\mathrm{HC}_{2} \mathrm{H}_{3} \mathrm{O}_{2} 0,5 \mathrm{~mol} \mathrm{~L}^{-1}$ $>$ Tampón pH 4,0 $\left(\mathrm{HC}_{2} \mathrm{H}_{3} \mathrm{O}_{2}+\mathrm{CH}_{3} \mathrm{COONa}\right)>$ solución de cloruro de bario $>\mathrm{CaCl}_{2} \mathrm{~mol} \mathrm{~L}^{-1}>\mathrm{CH}_{3} \mathrm{COONH}_{4} 38,5 \mathrm{~g} \mathrm{~L}^{-1}$, con un promedio de extracción de 25,01; 20,82; 11,18; 8,37 y $4,04 \mathrm{mg} \mathrm{kg}^{-1}$.

Para suelos neutros $\left(\mathrm{HC}_{2} \mathrm{H}_{3} \mathrm{O}_{2}\right.$ 0,5 $\left.\mathrm{mol} \mathrm{L}^{-1}\right)>($ Tampón pH 4,0 $\left(\mathrm{HC}_{2} \mathrm{H}_{3} \mathrm{O}_{2}+\mathrm{CH}_{3} \mathrm{COONa}\right)>$ (solución de cloruro de bario) $>$ $\left(\mathrm{CaCl}_{2} \mathrm{~mol} \mathrm{~L}^{-1}\right)>\left(\mathrm{CH}_{3} \mathrm{COONH}_{4} 38,5 \mathrm{~g} \mathrm{~L}^{-1}\right)$, con una extracción de 116,26; 83,67; 26,2; 24,95 y 15,19 $\mathrm{mg} \mathrm{kg}^{-1}$ y para suelos alcalinos, se halló la misma tendencia en extracción de silicio, con 148,36; 112,85; 24,26; 21,18 y $16,97 \mathrm{mg} \mathrm{kg}^{-1}$ (Tabla 2), resultados que indican que la menor cantidad de sillico extraído se presentó en los suelos ácidos, porque estos suelos, con arcillas tipo 2:1, son los más evolucionados y debido a las condiciones de precipitación y de temperatura, que oscilan entre 1.500 y $3.000 \mathrm{~mm}^{2}$ año $^{-1}$ y entre 30 y $40^{\circ} \mathrm{C}$, hay lixiviación del elemento a los horizontes subsuperficiales. Harley \& Gilkes, (2000) explican que la solución de suelo es un sistema abierto y la lixiviación y la difusión afectan a la disolución de productos durante el proceso de desgaste de minerales de silicato. Por otra parte, Stewart et al. (2001) explican que las tasas de agotamiento de minerales primarios y la formación de arcilla y óxido de metal secundario disminuyen progresivamente con la edad del suelo.

Con estos resultados fue constatado que el ácido acético a $0,5 \mathrm{~mol} \mathrm{~L}^{-1}$ y la solución tampón de $\mathrm{pH} 4,0$ tienen la capacidad de extraer silicio, que hace parte de compuestos insolubles, en los tres tipos de suelos. Además, a la alta acidez de las soluciones extractoras, que solubilizan el silicio, logran extraer los polímeros simples, que se encuentran en el suelo. Pereira et al. (2007) explican que el ácido acético también extrae silicio originario de polímeros simples, los cuales, son afectados por el cambio del pH de la solución y Korndörfer et al. (2004) indican que el ácido acético puede solubilizar pequeñas cadenas de Si polimerizado. Seron et al. (2007), al evaluar diferentes dosis de silicio y diferentes extractores encontraron que el ácido acético extrajo la mayor cantidad de silicio disponible. Pereira et al. (2004) encontraron que el ácido acético sobreestima el contenido de silicio en los suelos, principalmente, en los corregidos con cal agrícolas $\mathrm{y}$ en aquellos donde fueron aplicados fuentes ricas en aluminosilicatos.

Asimismo, las menores concentraciones de silicio fueron encontradas cuando se utilizaron las soluciones diluidas o 
Tabla 2. Contenido de Si disponible por cinco métodos de extracción en suelos ácidos, neutros y alcalinos, del departamento de Córdoba, Colombia.

\begin{tabular}{|c|c|c|c|c|c|}
\hline \multicolumn{6}{|c|}{ SUELOS ÁCIDOS } \\
\hline Suelos & M1 & M2 & M3 & M4 & \multirow[t]{2}{*}{ M5 } \\
\hline & \multicolumn{4}{|c|}{ - } & \\
\hline 1 & 12,35 & 27,13 & 4,89 & 18,94 & 17,49 \\
\hline 2 & 3,87 & 12,84 & 3,92 & 10,74 & 3,67 \\
\hline 3 & 9,84 & 33,38 & 2,81 & 25,87 & 12,84 \\
\hline 4 & 12,58 & 39,50 & 4,20 & 29,47 & 17,07 \\
\hline 5 & 8,85 & 31,97 & 4,43 & 31,92 & 12,30 \\
\hline 6 & 2,73 & 5,24 & 3,97 & 7,96 & 3,72 \\
\hline Promedio & 8,37 & 25,01 & 4,04 & 20,82 & 11,18 \\
\hline \multirow[t]{2}{*}{ Desvest } & 4,20 & 13,20 & 0,70 & 9,94 & 6,17 \\
\hline & \multicolumn{4}{|c|}{ SUELOS NEUTROS } & \\
\hline \multirow[t]{2}{*}{ Suelos } & M1 & M2 & M3 & M4 & M5 \\
\hline & \multicolumn{4}{|c|}{ 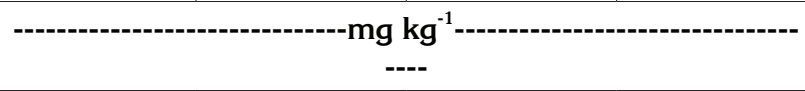 } & \\
\hline 1 & 19,54 & 114,92 & 10,18 & 84,74 & 16,45 \\
\hline 2 & 13,39 & 137,42 & 11,08 & 95,07 & 19,93 \\
\hline 3 & 15,77 & 47,78 & 6,56 & 37,11 & 14,75 \\
\hline 4 & 48,06 & 198,31 & 38,18 & 149,78 & 47,69 \\
\hline 5 & 15,16 & 41,81 & 7,22 & 28,45 & 17,58 \\
\hline 6 & 37,78 & 157,28 & 17,88 & 106,87 & 40,81 \\
\hline Promedio & 24,95 & 116,26 & 15,19 & 83,67 & 26,20 \\
\hline \multirow[t]{2}{*}{ Desvest } & 14,43 & 61,80 & 11,97 & 45,29 & 14,25 \\
\hline & \multicolumn{5}{|c|}{ SUELOS ALCALINOS } \\
\hline \multirow[t]{2}{*}{ Suelos } & M1 & M2 & M3 & M4 & M5 \\
\hline & \multicolumn{4}{|c|}{ 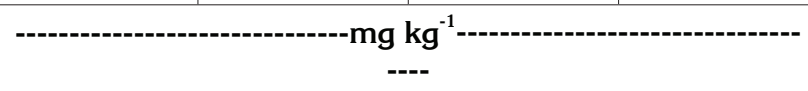 } & \\
\hline 1 & 22,34 & 135,88 & 14,27 & 68,27 & 23,78 \\
\hline 2 & 16,57 & 132,82 & 11,89 & 111,04 & 20,47 \\
\hline 3 & 16,07 & 200,89 & 13,19 & 173,30 & 23,06 \\
\hline 4 & 25,59 & 147,65 & 10,64 & 66,93 & 28,62 \\
\hline 5 & 16,93 & 109,36 & 12,51 & 103,37 & 20,53 \\
\hline 6 & 29,56 & 163,59 & 39,30 & 154,19 & 29,08 \\
\hline Promedio & 21,18 & 148,36 & 16,97 & 112,85 & 24,26 \\
\hline Desvest & 5,59 & 31,34 & 11,01 & 43,71 & 3,80 \\
\hline
\end{tabular}

M1: $\mathrm{CaCl}_{2} \mathrm{~mol} \mathrm{~L}^{-1}$, M2: $\mathrm{HC}_{2} \mathrm{H}_{3} \mathrm{O}_{2}$ 0,5mol L-1, M3: $\mathrm{CH}_{3} \mathrm{COONH}_{4} 38,5 \mathrm{~g} \mathrm{~L}^{-1}$, M4: Tampón pH 4,0 $\left(\mathrm{HC}_{2} \mathrm{H}_{3} \mathrm{O}_{2}+\mathrm{CH}_{3} \mathrm{COONa}\right)$, M5: solución de $\mathrm{BaCl}_{2}$. Desvest: desviación estándar. 
salinas, como el cloruro de calcio $0,01 \mathrm{~mol} \mathrm{~L}^{-1}$ y acetato de amonio, que extraen la menor cantidad de silicio disponible, en estos suelos. La pequeña cantidad de silicio extraído por estos métodos está directamente relacionada con el $\mathrm{pH}$ de las soluciones, porque a este $\mathrm{pH}$, no hay solubilización de compuestos insolubles. Berthelsen et al. (2001) indican que el agente de extracción más débil después del agua es el $\mathrm{CaCl}_{2}$, que solo extrae la fracción de Si fácilmente soluble y Pereira et al. (2007), en un estudio de comparación de métodos de extracción de silicio en diferentes clases de suelos en Australia, encontraron que los extractores tienden a solubilizar el Si de diferentes componentes de la matriz del suelo.

Al analizar las correlaciones entre los contenidos de silicio extraído, entre los métodos estudiados y el método de re- ferencia cloruro de calcio $0,01 \mathrm{~mol} \mathrm{~L}^{-1}$ en los suelos ácidos (Figura 1), se observa que el coeficiente de correlación (r) fue altamente significativo $(0,99 * *)$, cuando se comparó la extracción de silicio con el método de agua modificado y el método de referencia. Resultados que son diferentes cuando fueron evaluados los extractores, ácido acético $0,5 \mathrm{~mol} \mathrm{~L}^{-1}$, solución tampón de pH 4,0 y el acetato de amonio 38,5g $\mathrm{L}^{-1}$, que presentaron coeficientes de correlación no significativos, debido a la capacidad de extraer silicio de compuestos insolubles. Xu et al. (2001) registraron que los extractantes ácidos, como acetato sódico y ácido cítrico, extraen las mayores cantidades de Si en suelos calcáreos, que son disueltos a partir de silicatos cálcicos, solubles en ácidos, pero no absorbidos fácilmente por las plantas.
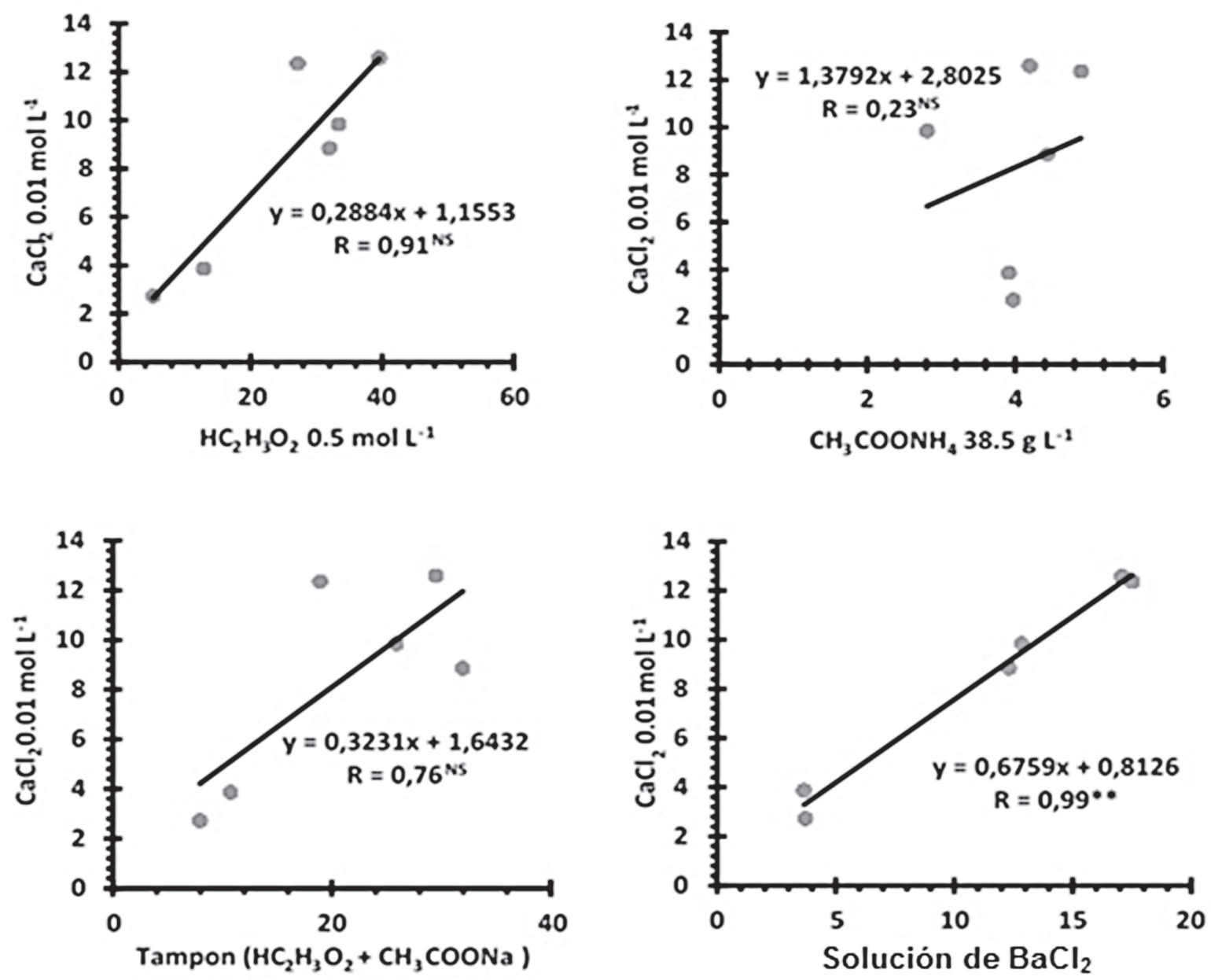

Figura 1. Relación entre el contenido de Si disponible, extraído por cloruro de calcio y por diferentes métodos de extracción, en suelos ácidos, neutros y alcalino, del departamento de Córdoba, Colombia. 
Estas diferencias entre el método de referencia y agua modificado, se pueden explicar, porque el método de agua modificada solubiliza el silicio, que se encuentra en la fase coloidal del suelo. Además, estas soluciones tienen en común el mismo catión índice, que hace que funcioné como buen extractor de este elemento, por lo tanto, la cantidad de $1 \mathrm{~mL}$ de $\mathrm{BaCl}_{2}$ al $10 \%$ ocasiona una baja capacidad de extracción $y$, consecuentemente, no hay solubilización de los complejos de silicio formados por óxidos de hierro y de aluminio y pequeñas cadenas poliméricas de este elemento. Savenko (2015), al determinar la cantidad de Si disuelto en los suelos, halló que alcanza 6 al 20\% de la sílice total, en la fase sólida.
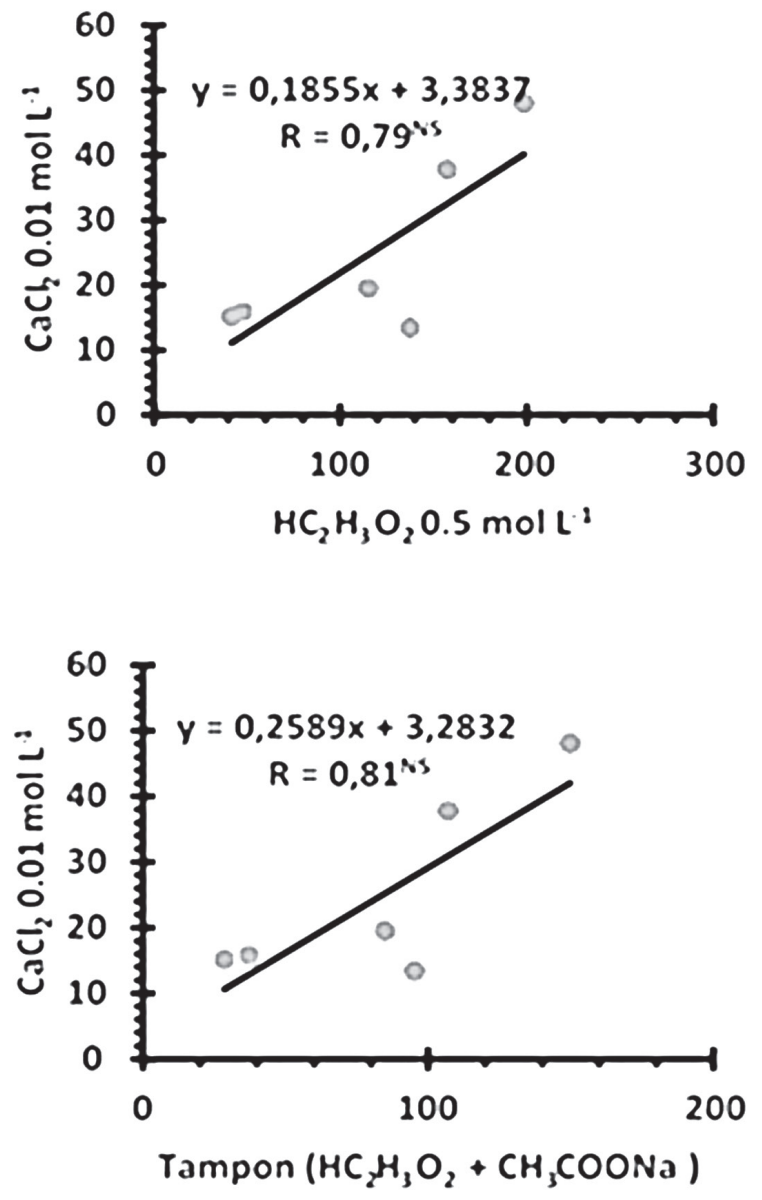

Para los suelos neutros, también el extractante que presentó el mayor coeficiente de correlación fue el método solución de cloruro de bario, con referencia al método de cloruro de calcio $0,01 \mathrm{~mol} \mathrm{~L}^{-1},\left(r=0,97^{*}\right)$, seguido por el acetato de amonio $38,5 \mathrm{mg} \mathrm{L}^{-1}\left(\mathrm{r}=0,92^{*}\right)$. Esto resultados indican que estos extractores tienen la capacidad de extraer el silicio disponible que se encuentra en el suelo, en magnitudes similares al método de referencia, por lo tanto, estos métodos pueden ser considerados como alternativa para el proceso de extracción de silicio disponible en suelos neutros (Figura 2). Ferreira et al. (2010) recomiendan el uso de $\mathrm{CaCl}_{2}$ y ácido acético para la extracción de silicio, en suelos con altos contenidos de acilla.
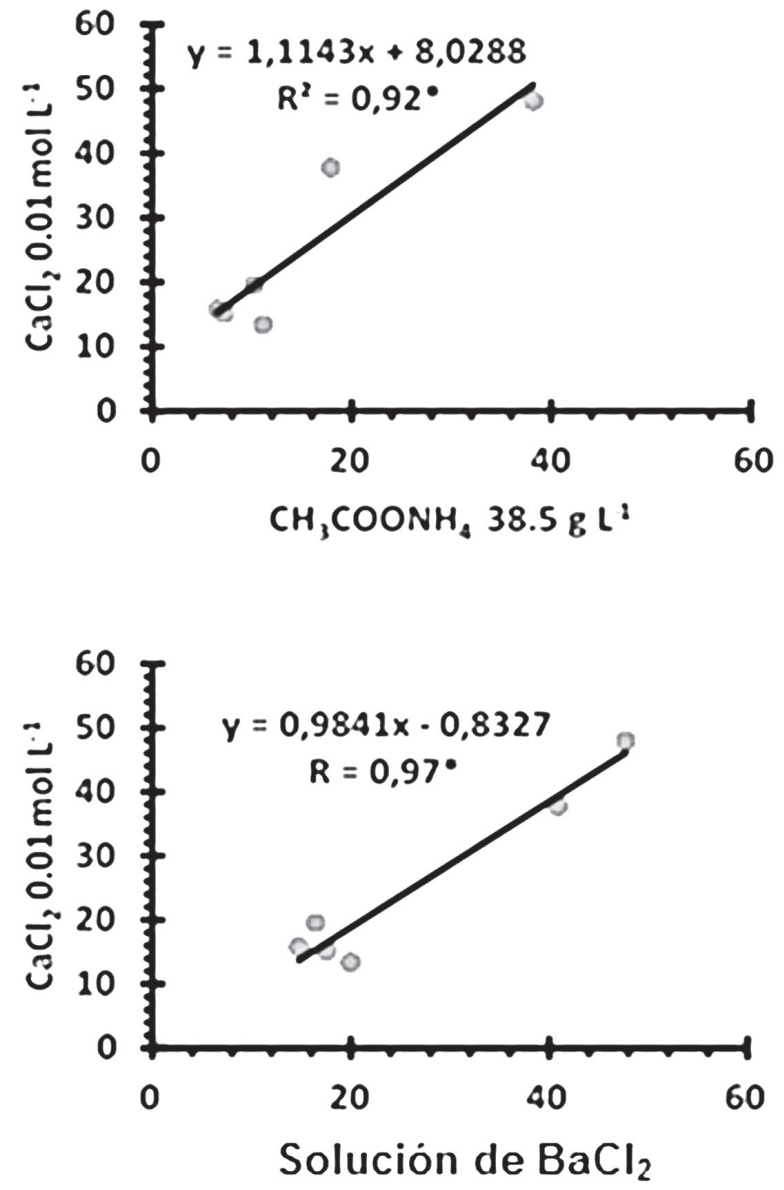

Figura 2. Relación entre el contenido de Si disponible, extraído por cloruro de calcio $0,01 \mathrm{~mol} \mathrm{~L}^{-1}$ y por diferentes métodos, en suelos neutros, del departamento de Córdoba, Colombia. 
En la figura 3, se observa que en los suelos alcalinos también se presentó una correlación positiva entre el método de referencia y el método de agua modificada, con una correlación significativa $(r=0,93)$, mientras que para el método de ácido acético $(r=0,09)$, acetato de amonio $(r=0,71)$ y tampón $(r=0,14)$ presentaron coeficientes de correlación no significativos. El bajo coeficiente de correlación para estos métodos, se puede explicar por la alta extracción de silicio originarios de polímeros, que hacen parte de la estructura de minerales de arcilla 2:1, donde los cambios de $\mathrm{pH}$ consiguen solubilizar el silicio, que es insoluble en estas condiciones
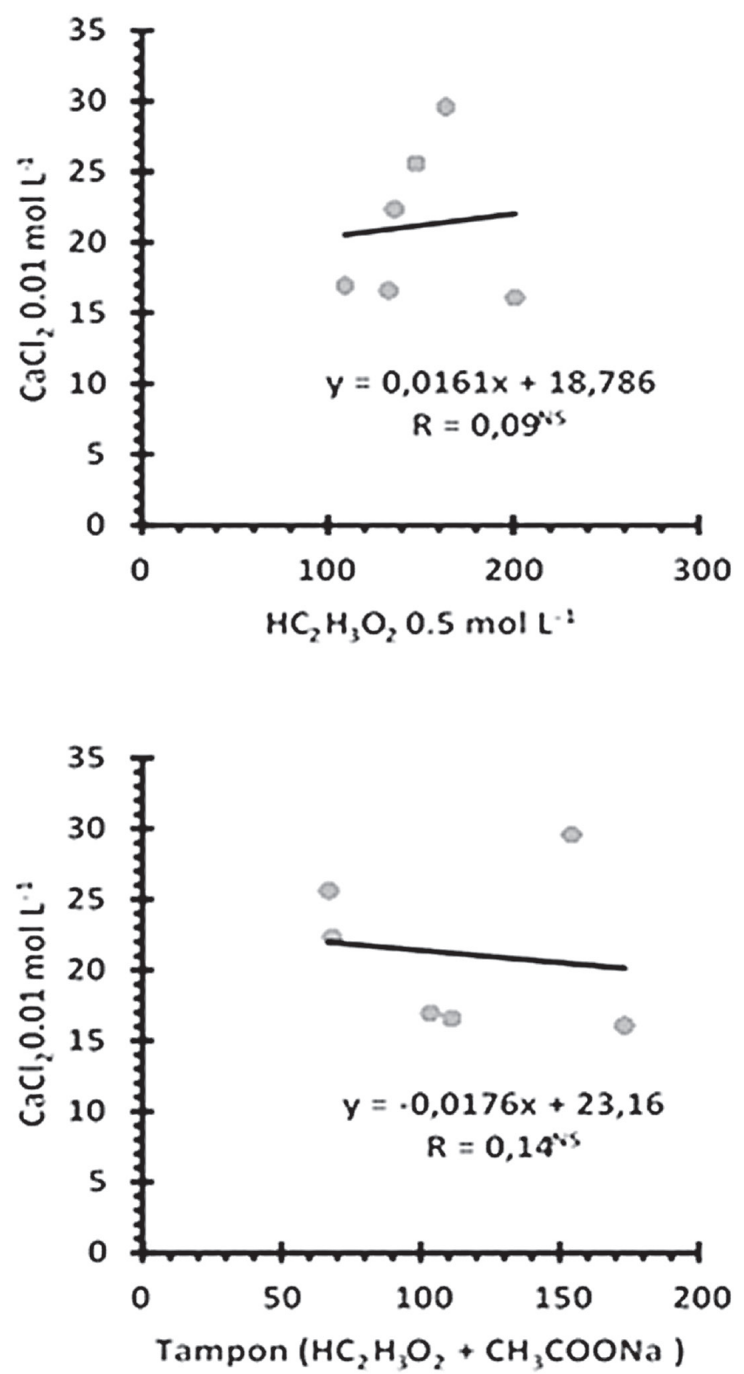

químicas de suelos. Korndörfer et al. (2004) explican que el ácido acético es un ácido débil, capaz de extraer Si disponible para las plantas, sin atacar, de forma muy agresiva, los minerales de arcilla, con capacidad de solubilizar pequeñas cadenas de Si polimerizadas. Korndörfer et al. (2001) exponen que la extracción de silicio con ácido acético 0,5N fue desarrollado, específicamente, en estudios realizados sobre los suelos orgánicos y minerales de la región Sur de Florida que, por lo general, reflejan bajo contenido de arcilla, $\mathrm{Al}$ y Fe, sin conocer si esta solución era apropiada para otros tipos de suelo.
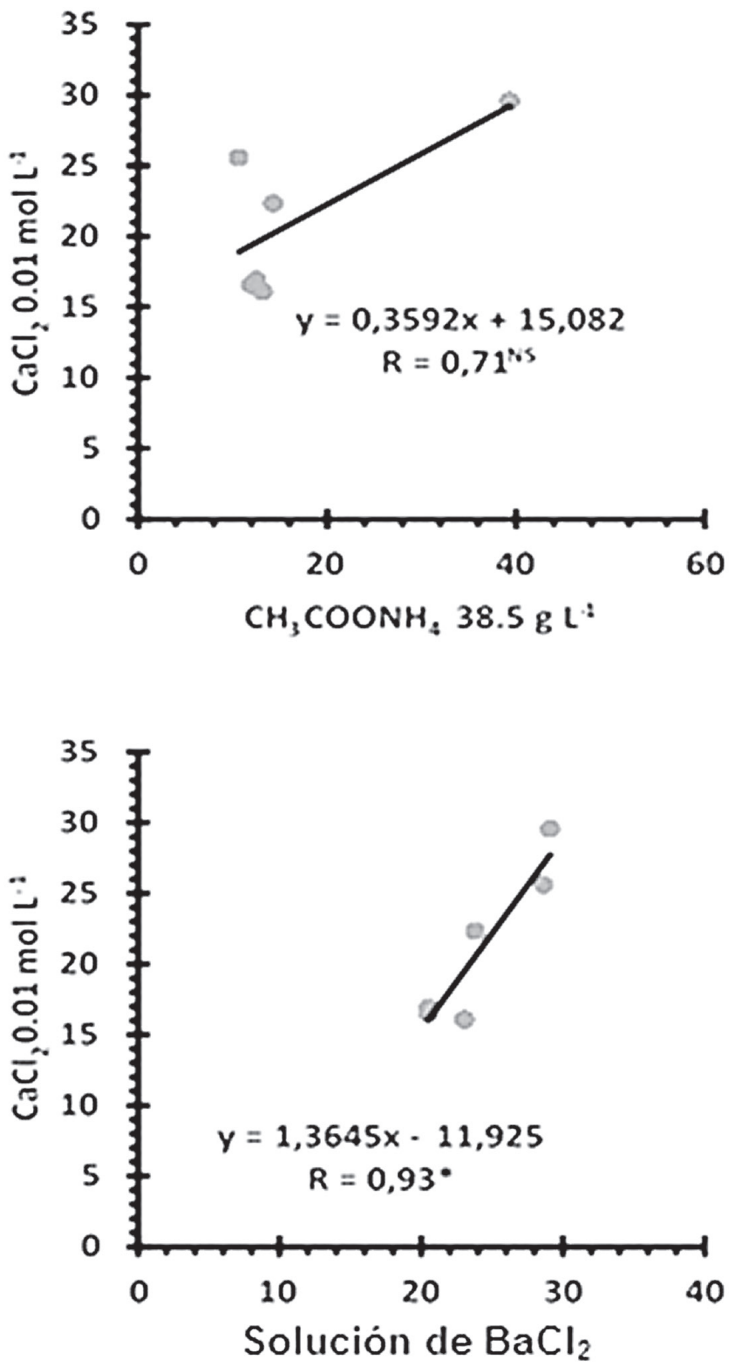

Figura 3. Relación entre el contenido de Si disponible, extraído por cloruro de calcio y por diferentes métodos, en suelos alcalinos, del departamento de Córdoba-Colombia. 
Además, los resultados encontrados entre el método de una solución de cloruro de bario y el método cloruro de calcio $0,01 \mathrm{~mol} \mathrm{~L}^{-1}$, se puede explicar, porque el poder de extracción de estas soluciones está en función de las interacciones catión índice y el elemento a desplazar (Si) y, químicamente, la reacciones que ocurren logran un equilibrio similar, con el cual, hay solubilización del silicio disponible del suelo. Wang et al. (2004) explican que la acidez y los aniones podrían impactar, de forma aditiva, la liberación de Si de los suelos. Asimismo, comparten características físico químicas parecidas, como la configuración electrónica, radio atómico, características no metálicas y también la atracción por sus estados de oxidación, particulares que influyen en estos extractores, para extraer el silicio disponible.

Estos resultados difieren con los estudios de correlación realizados por Pereira et al. (2007), en los cuales, el acetato de amonio $38,5 \mathrm{~g} \mathrm{~L}^{-1}$ presentó mejores coeficientes de correlación que la extracción con agua, lo que indica que este extractor es más confiable después del cloruro de calcio $0,01 \mathrm{~mol} \mathrm{~L}^{-1}$.

Por otra parte, el extractor ácido acético $0,5 \mathrm{~mol} \mathrm{~L}^{-1}$ fue el extractor químico que presentó los coeficientes de correlación más bajos para los suelos neutros y alcalinos. Resultados que demuestran la capacidad de extraer silicio de compuesto de baja solubilidad, que se encuentran en los suelos, formando cadenas poliméricas de silicio, que promueven la polimerización y la formación de complejos de aluminosilicatos y, como consecuencia, se puede presentar una sobrestimación del silicio, disponible para las plantas. Xu et al. (2001) atribuyeron la disolución de silicato de calcio en suelos calcáreos, al alto contenido de silicio extraído con solución extractora de ácido (pH 4) y, de acuerdo con Wang et al. (2004), la acidez y los aniones podrían impactar, de forma aditiva, la liberación de Si de los suelos. Gascho (2001) indica que no hay extractante universal que sea adecuado para determinar el Si disponible en todos los tipos de materiales y para todos los suelos y condiciones del suelo.

Para confirmar los resultados encontrados, se realizaron contrastes al 5\% de probabilidad, con el objetivo de comparar las diferencias estadísticas entre todos los métodos de extracción de silicio disponible en suelos ácidos, neutros y alcalinos. En los suelos ácidos, se evidenció que existen diferencias altamente significativas en los contrastes $\mathrm{C} 1, \mathrm{C} 2$, $\mathrm{C} 3$, C5 y C6, pero para el contraste $\mathrm{C} 4, \mathrm{CaCl}_{2} 0,01 \mathrm{~mol} \mathrm{~L}^{-1}$ vs solución de cloruro de bario, no hubo diferencias estadísticas significativas (Tabla 3). Resultados similares fueron encontrados en las correlaciones, por lo anterior estos métodos son alternativos.

Para los suelos neutros, los contrastes C1, C2, C3, C5 y C6 presentan diferencias altamente significativas y el contraste C4 no evidenció diferencias estadísticas. Además, para los suelos alcalinos, se encontró que los contrastes C4 y C2, no presentaron diferencias estadísticas. Babu et al. (2013) han detectado que las cantidades de Sí determinadas, utilizando diferentes extractores, varían significativamente en suelo de Estados Unidos.

Con estos resultados, se constató que en los tres tipos de suelos se presentó extracción de silicio en magnitudes, estadísticamente iguales, entre el método de referencia y el método de solución de cloruro de bario, pero para los suelos alcalinos el $\mathrm{CH}_{3} \mathrm{COONH}_{4}$ puede ser también una alternativa

Tabla 3. Contrastes para el contenido de silicio disponible, por cinco métodos de extracción, en suelos ácidos, neutros y alcalinos, del departamento de Córdoba- Colombia.

\begin{tabular}{|c|c|c|c|c|}
\hline \multirow{2}{*}{ Contrastes } & \multirow{2}{*}{ Métodos } & \multicolumn{3}{|c|}{ Contenido de Si disponible } \\
\hline & & Ácidos & Neutros & Alcalinos \\
\hline C1 & $\mathrm{CaCl}_{2}$ vs $\mathrm{HC}_{2} \mathrm{H}_{3} \mathrm{O}_{2}$ & $16.64 * *$ & $91.31 * *$ & $127.18 * *$ \\
\hline $\mathrm{C} 2$ & $\mathrm{CaCl}_{2}$ vs $\mathrm{CH}_{3} \mathrm{COONH}_{4}$ & $-4.33 * *$ & $-9.76 * *$ & $-4.21^{\mathrm{NS}}$ \\
\hline $\mathrm{C} 3$ & $\mathrm{CaCl}_{2}$ vs Tampón $\left(\mathrm{HC}_{2} \mathrm{H}_{3} \mathrm{O}_{2}+\mathrm{CH}_{3} \mathrm{COONa}\right)$ & $12.44 * *$ & $58.72 * *$ & $91.67 * *$ \\
\hline $\mathrm{C} 4$ & $\mathrm{CaCl}_{2}$ vs solución de $\mathrm{BaCl}_{2}$ ) & $2.81^{\mathrm{NS}}$ & $1.25^{\mathrm{NS}}$ & $3.24^{\mathrm{NS}}$ \\
\hline $\mathrm{C} 5$ & $\mathrm{CaCl}_{2}$ vs $^{\mathrm{HC}_{2} \mathrm{H}_{3} \mathrm{O}_{2}}+\mathrm{CH}_{3} \mathrm{COONH}_{4}$ & $12.31 * *$ & $81.54 * *$ & $122.97 * *$ \\
\hline C6 & $\mathrm{HC}_{2} \mathrm{H}_{3} \mathrm{O}_{2}+\mathrm{CH}_{3} \mathrm{COONH}_{4}$ vs solución de $\mathrm{BaCl}_{2}$ & $-6.68 * *$ & $-79.04 * *$ & $-116.47 * *$ \\
\hline
\end{tabular}

C1: $\mathrm{CaCl}_{2}$ Vs $\mathrm{HC}_{2} \mathrm{H}_{3} \mathrm{O}_{2} \mathrm{H}_{2} \mathrm{O}$, C2: $\mathrm{CaCl}_{2} \mathrm{Vs} \mathrm{CH}_{3} \mathrm{COONH}_{4}, \mathrm{C} 3: \mathrm{CaCl}_{2}$ Vs Tampón $\left(\mathrm{HC}_{2} \mathrm{H}_{3} \mathrm{O}_{2}+\mathrm{CH}_{3} \mathrm{COONa}\right.$ C4: CaCl Vs solución de cloruro de bario; C5: $\mathrm{CaCl}_{2} \mathrm{Vs} \mathrm{HC}_{2} \mathrm{H}_{3} \mathrm{O}_{2}+\mathrm{CH}_{3} \mathrm{COONH}_{4}$ C6: $\mathrm{HC}_{2} \mathrm{H}_{3} \mathrm{O}_{2}+\mathrm{CH}_{3} \mathrm{COONH}_{4} \mathrm{Vs} \mathrm{H}_{2} \mathrm{O}$ (Modificado); NS: no significativo , * Significativo al $5 \%$ y **significativo al $1 \%$ 
de uso en la estimación de silicio. Snyder (2001) exponen que la extracción de silicio de los suelos con acetato solo extrae el silicio disuelto y algunos de los enlaces de silicio intercambiable de los suelos; igualmente, Barbosa et al. (2000) indican que, para conocer la necesidad de fertilización con silicio, es necesario desarrollar métodos eficientes de análisis de silicio disponible en los suelos, en las plantas y en los fertilizantes. Sauer et al. (2006) mencionaron que hay poco conocimiento sobre la extracción de Si y como resultado hay un creciente interés en seleccionar un método de extracción química, que pueda proporcionar estimaciones confiables y reproductible.

De la presente investigación, se puede destacar que la disponibilidad de silicio en los suelos se presentó en el siguiente orden: suelos alcalinos $>$ neutros $>$ ácidos. El ácido acético $0,5 \mathrm{~mol} \mathrm{~L}^{-1}$ fue el que más extrajo silicio en los suelos ácidos, neutros y alcalinos y el acetato de amonio fue el que presentó la menor extracción, en los tres tipos de suelos. Además, los resultados de las pruebas estadísticas realizadas indicaron que el método, con una solución de cloruro de bario, tiene la misma tendencia en la extracción de silicio disponible en los suelos ácidos, neutros y alcalinos, que el método de referencia $\mathrm{CaCl}_{2}$ 0,01 $\mathrm{mol} \mathrm{L}^{-1}$, a excepción del método de acetato de amonio $38,5 \mathrm{~g} \mathrm{~L}^{-1}$, que presenta igual comportamiento que el método de referencia en los suelos alcalinos.

Agradecimientos: Los autores agradecen al centro de investigaciones de la Universidad de Córdoba (CIUC), por el apoyo financiero para la ejecución del proyecto "Cuantificación de fracciones granulométricas y elementos nutricionales utilizando diferentes extractantes en suelos del departamento de Córdoba", además, a los analistas químicos del laboratorio de Suelos y Aguas de la Facultad de Ciencias Agrícolas. También, se recomienda seguir con el estudio de métodos analíticos para elementos benéficos, ya que son pocos los estudios realizados en nuestro país. Conflictos de intereses: El manuscrito fue preparado y revisado con la participación de todos los autores, quienes declaramos que no existe conflicto de intereses que ponga en riesgo la validez de los resultados presentados.

\section{BIBLIOGRAFÍA}

1. BARBOSA FILHO, M.P.; SNYDER, G.H.; PRABHU, A.S.; DATNOFF, L.E.; KORNDÖRFER, G.H. 2000. Importância do silício para a cultura do arroz. Piracicaba: Potafos. Encarte técnico. 89p.

2. BABU, T.; DATNOFF, L.; YZENAS, J.; TUBANA. B. 2013. Silicon status of Louisiana soils grown to different field crops. In: ASA-CSSA-SSSA International Annual Meetings. November 3-6, 2013. Tampa, FL.
3. BERTHELSEN, S.; KORNDÖRFER, G. 2005. Methods for $\mathrm{Si}$ analysis in plant, soil and fertilizers. In: Silicon in agriculture conference, 3. Proceedings. Uberlândia: Universidade Federal de Uberlândia. p.85-90.

4. BERTHELSEN, S.; NOBLE, A.D.; GARSIDE, A.L. 2001. Silicon research down under: past, present and future. In: Datnoff, L.E.; Snyder, G.H.; Korndörfer, G.H. (eds). Silicon Deposition in Higher Plants. Silicon in Agriculture. Elsevier Science. p.241-255.

5. CONDRON, L.M.; NEWMAN, S. 2011. Revisiting the fundamentals of phosphorus fractionation of sediments and soils. J. Soil Sediment. 11:830-840.

6. FAUTEUX, F.; REMUS-BOREL, W.; MENZIES, J.G.; BÉLANGER, R.R. 2005. Silicon and plant disease resistance against pathogenic fungi. FEMS Microbiol Lett. 249:1-6.

7. FERREIRA, M.I.; SANTOS, G.R.; OSCAR, N.J. 2010. Avaliação de métodos de extração de silício e fósforo em amostras de solos. Rev. Caatinga, Mossoró. 23(4):75-83.

8. GASCHO, G.J. 2001. Silicon Sources for Agriculture. In: Datnoff, L.E.; Snyder, G.H.; Korndörfer, G.H. (eds.). Silicon in Agriculture. Elsevier Science B.V. Amsterdam. The Netherlands. 424p.

9. HARLEY, A.D.; GILKES, R.J. 2000. Factors influencing the release of plant nutrient elements from silicate rock powders: A geochemical overview. Nutr. Cycl. Agroecosyst. 56:11-36.

10. HAVLIN, L.; BEATON, J.; TISDALE, S.; NELSON, W. 2005. Soil fertility and fertilizers. 7. ed. Upper Saddle River: Pearson Education. 515p.

11. INSTITUTO GEOGRÁFICO AGUSTÍN CODAZZI -IGAC. 2006. Métodos analíticos del laboratorio de suelos. VI Edición. Bogotá, Subdirección de Agrología. 499p.

12. KORNDÖRFER, G.; COELHO, N.; SNYDER, G.; MIZUTANI, C. 1999. Avaliação de métodos de extração de silício para solos cultivados com arroz de sequeiro. Rev. Bras. Cienc. Solo. 23(1):101-106.

13. KORNDÖRFER, G.; PEREIRA, H.; NOLLA, A. 2004. Análise de silício: solo, planta e fertilizante. 2. ed. Uberlândia: GPSi: ICIAG: UFU. (UFU. ICIAG. Bol. Técnico 2. 34p. 
14. KORNDÖRFER, G.H.; SNYDER, G.H.; ULLOA, M.; POWELL, G.; DATNOFF, L.E. 2001. Calibration of soil and plant silicon analysis for rice production. J. Plant Nutr. 24(7):1071-1084.

15. LIMA, R.L.; DAROUB, S.H.; RICE, R.W.; SNYDER, G.H. 2003. Comparison of three soil test methods for estimating plant-available silicon. Communications in Soil SciencE and Plant Analysis. 34(15-16):20592071.

16. LIMA, M.A.; CASTRO, V.F.; VIDAL, J.B.; ENÉAS-FILHO, J. 2011. Aplicação de silício em milho e feijãodecorda sob estresse salino. Rev. Ciência Agronômica. 42(2):398-403.

17. PEREIRA, H.; KORNDÖRFER, S.; GASPAR, H.; VIDAL, A.; DE AQUINO, C.; SARTORI, M. 2004. Silicon sources for rice crop. Scientia Agrícola. 61(5):522528.

18. PEREIRA, H.; BARBOSA, N.C.; CARNEIRO, M.; AURÉLIO, C.; KORNDÖRFER, G. 2007. Avaliação de fontes e de extratores de silício no solo. Pesquisa Agropecuária Bras. 42(2):239-247.

19. PULZ, A.L.; CRUSCIOL, C.; COSTA, A.; LEMOS, L.B.; SORATTO, R.P. 2008. Influência de silicato e calcário na nutrição, produtividade e qualidade da batata sob deficiência hídrica. Rev. Bras. Cienc Solo. 32(4):1651-1659.

20. SAVENKO, A.V. 2015. The possibility of phosphatization of silicates in the supergene zone. Geochem. International. 53(1):87-94.

21. SAUER, D.; SACCONE, L.; CONLEY, D.J.; HERRMANN, L.; SOMMER, M. 2006. Review of methodologies for extracting plant-available and amorphous $\mathrm{Si}$ from soils and aquatic sediments. Biogeochemistry. 80:89-108.

22. SERON, P.H.; CABRAL, B.N.; CARBONE, C.M.A.; KORNDÖRFER, G.H. 2007. Avaliação de fontes e de extratores de silício no solo. Pesq. Agropec. Bras. 42(2):239-247.

23. SHETTY, R.; FRETTE, X.; JENSEN, B.; SHETTY, N.P.; JENSEN, J.D.; ØRGENSEN, H.J.L.; NEWMAN, M.A.; CHRISTENSEN, L.P. 2011. Silicon-induced changes in antifungal phenolic acids, flavonoids, and key phenylpropanoid pathway genes during the interaction between miniature roses and the biotrophic pathogen Podosphaera pannosa. Plant Physiology. 157:2194-2295.

24. SILVA, F.R.; FERREYRA H., F.F. 1998. Avaliação de extratores de boro em solos do Estado do Ceará. Rev. Bras. Cienc. Solo. 22:471-478.

25. SNYDER, G. 2001. Methods for silicon analysis in plants, soils, and fertilizers. In: Datnoff, L, Snyder, G. H.; Korndörfer, G. H. (Ed.). Silicon in agriculture. Amsterdam: Elsevier.p.185-196.

26. STEWART, B.W.; CAPO, R.C.; CHADWICK, O.A. 2001. Effects of rainfall on weathering rate, base cation provenance, and $\mathrm{Sr}$ isotope composition of Hawaiian soils. Geochim. Cosmochim. Acta. 65:1087-1099.

27. VIDAL, A. 2003. Fontes de silício para a cultura do arroz. Uberlândia, Universidade Federal de Uberlândia. 34p.

28. XU, G.; ZHAN, X.; CHUNHUA, L.; BAO, S.; LIU, X.; CHU, T. 2001. Assessing methods of available silicon in calcareous soils. Commun.in Soil Sci. Plant Anal. 32:787-801.

29. WANG, J.J.; DODLA, S.K.; HENDERSON, R.E. 2004. Soil silicon extractability with seven selected extractants in relation to colorimetric and ICP determination. Soil Science. 169:861-870.

Recibido: Octubre 3 de 2016

Aceptado: Agosto 8 de 2017

Cómo citar:

Combatt C., E.; Palacio B., D.; Mercado L., J. 2017. Extractores químicos en la determinación de silicio disponible en suelos ácidos, neutros y alcalinos. Rev. U.D.C.A Act. \& Div. Cient. 20(2): 329-339. 